International Journal of Pure and Applied Mathematics

Volume 97 No. 4 2014, 515-525

ISSN: 1311-8080 (printed version); ISSN: 1314-3395 (on-line version)

url: http://www.ijpam.eu

doi: http://dx.doi.org/10.12732/ijpam.v97i4.11

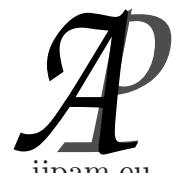

ijpam.eu

\title{
RADIO NUMBER FOR CROSS PRODUCT $\boldsymbol{P}_{n}\left(\boldsymbol{P}_{2}\right)$
}

\author{
Chahn Yong Jung ${ }^{1}$, Waqas Nazeer ${ }^{2}$, Saima Nazeer ${ }^{3}$, \\ Arif Rafiq ${ }^{4}$ and Shin Min Kang ${ }^{5} \S$ \\ ${ }^{1}$ Department of Business Administration \\ Gyeongsang National University \\ Jinju, 660-701, KOREA \\ ${ }^{2,4}$ Department of Mathematics \\ Lahore Leads University \\ Lahore, 54810, PAKISTAN \\ ${ }^{3}$ Department of Mathematics \\ Lahore College for Women University \\ Lahore, 54600, PAKISTAN \\ ${ }^{5}$ Department of Mathematics and RINS \\ Gyeongsang National University \\ Jinju, 660-701, KOREA
}

\begin{abstract}
A Radio labeling of the graph $G$ is a function $g$ from the vertex set $V(G)$ of $G$ to $\mathbb{N} \cup\{0\}$ such that $|f(u)-f(v)| \geq \operatorname{diam}(G)+1-d_{G}(u, v)$, where $\operatorname{diam}(G)$ and $d_{G}(u, v)$ are diameter and distance between $u$ and $v$ in graph $G$, respectively. The radio number $\operatorname{rn}(G)$ of $G$ is the smallest number $k$ such that $G$ has radio labeling with $\max \{f(v): v \in V(G)\}=k$. We investigate radio number for the cross product of $P_{n}$ and $P_{2}$.
\end{abstract}

AMS Subject Classification: 05C12, 05C15, 05C 78

Key Words: channel assignment, radio labeling, radio number, cross product

Received: August 28, 2014

(C) 2014 Academic Publications, Ltd. url: www.acadpubl.eu

${ }^{\S}$ Correspondence author 


\section{Introduction}

In 1980, Hale [5] presented the idea for radio frequency assignment problems. Later in 2001, Chartrand et al. [2] applied this idea for assignment of channels to FM radio station. These assignment have been made on the fact that frequencies need to be assigned to the channels such that there is minimum interference. The geographically closed radio stations should be assigned different frequencies to avoid interference.

The interference graph is developed to solve the channels assignment problems by converting an assignment of channels in to graph labeling. In interference graph, there is an edge between two vertices if the corresponding transmitter have major interference. It is assumed that the distance between vertices is two if two transmitters have minor interference in interference graph and if the vertices are at distance three or beyond then there is no interference between transmitters.

In other words, the adjacent vertices represent the very close transmitters and those vertices which are at distance two apart represents close transmitters. A pair of transmitters which has small interference must receive different channels and two transmitters which has large interference must receive channels that are at least two apart are suggested in [12] by Robert.

Motivated through this problem Griggs and Yeh [4] relates the channels with non-negative integer by introducing $L(2,1)$-labeling, which is defined as follows:

Definition 1.1. A distance two labeling (or $L(2,1)$-labeling) of a graph $G=(V(G), E(G))$ is a function $g$ from vertex set $V(G)$ to the set of nonnegative integers such that the following conditions are satisfied:

(1) $|g(u)-g(v)| \geq 2$ if $d(u, v)=1$,

(2) $|g(u)-g(v)| \geq 1$ if $d(u, v)=2$.

The difference between the largest and the smallest label assigned by $g$ is called the span of $g$ and the minimum span over all $L(2,1)$-labeling of $G$ is called the $\lambda$-number of $G$, denoted by $\lambda(G)$. The $L(2,1)$-labeling has explored in the past two decades by many researchers like Chang and Kuo [1], Georges and Mauro [3], Sabaki [13], Vaidya and Bantva [14], Vaidya et al. [16], Wang [17] and Yeh [18], [19].

But as time passed, practically it has been observed that the interference among transmitters might go beyond two levels. Radio labeling extends the number of interference level considered in $L(2,1)$-labeling from two to largest possible interference among transmitter, i.e. the diameter of $G$ which is defined 
as follows:

Definition 1.2. The diameter of a graph is denoted by $\operatorname{diam}(G)$ and defined as the maximum distance between any two vertices, that is, $\operatorname{diam}(G)=$ $\max \{d(u, v) ; u, v \in G\}$.

Here $d(u, v)$ is distance between $u$ and $v$ which is defined as follows:

Definition 1.3. Let $G$ be a connected graph, the distance $d(u, v)$ between any pair of vertices $u, v$ is the length of the shortest path between them.

Motivated through the problem of channel assignment of FM radio stations Chartrand et al. [2] introduced the concept of radio labeling which also known as Multi-level distance labeling of graph as follows:

Definition 1.4. A radio labeling which is also known as multilevel distance labeling of $G$ is a function $g: V(G) \rightarrow \mathbb{N} \cup\{0\}$ such that the inequality $|g(u)-g(v)| \geq \operatorname{diam}(G)+1-d(u, v)$ holds for any pair of distinct vertices $u, v$. The span of $g$ is the difference of the largest and the smallest channels used, $\max _{u, v \in V(G)}\{g(v)-g(u)\}$. The radio number of $G$ is denoted by $\operatorname{rn}(G)$ and is defined as the minimum span of radio labeling of $G$.

Note that when $\operatorname{diam}(G)$ is two than radio labeling and distance two labeling are identical. The radio labeling is studied in the past decade by many researches like Liu [6], Liu and Xie [7], [8], Liu and Zhu [9] and Vaidya and Vihol [15]. Moreover, the radio number for path and cycles was determined in [9], for the square of paths was investigated by Liu and Xie [8], for the square of a cycle [7]. Radio Number for generalized prism graph was studied in [10] and a generalized gear graph was discussed in [11], where lower bound of radio number is determined. Radio labeling for some cycle related graphs are studied by Vadiya and Vihol [15].

In this paper, we completely determine the radio number of cross product for $P_{n}$ and $P_{2}$. Through our this discussion, the order of $P_{n}\left(P_{2}\right)$ is $2 n$. In Theorem 2.2 we determine the radio number for $P_{n}\left(P_{2}\right)$. Theorem 2.3 and Theorem 2.5 give us lower and upper bound for radio number of $P_{n}\left(P_{2}\right) ; n \geq 3$, and finally in Theorem 2.6 we get $r n\left(P_{n}\left(P_{2}\right)\right)=n^{2}-n+1$.

\section{Main Results}

The cross product of graphs $G$ and $H$ denoted by $G(H)$, is the graph with the vertex set $V(G) \times V(H)=\{(u, v): u \in V(G), v \in V(H)\}$, where $(u, x)$ 
is adjacent to $(v, y)$ whenever (i) $u_{1}=v_{1}$ and $u_{2} \leftrightarrow v_{2}$ or (ii) $u_{1} \rightarrow v_{1}$ and $u_{2} \leftrightarrow v_{2}$.

CASE 1: For $P_{n}\left(P_{2}\right)$, when $n$ is odd, let $v_{0}$ and $u_{0}$ be the centers. Let $v_{1}, v_{2}, \cdots, v_{\frac{n-1}{2}}$ be the vertices on the left side and $v_{1}^{\prime}, v_{2}^{\prime}, \cdots, v_{\frac{n-1}{2}}^{\prime}$ be the vertices on the right side with respect to center $v_{0}$ and $u_{1}, u_{2}, \cdots, u_{\frac{\mathrm{n}-1}{2}}$ be the vertices on the left side and $u_{1}^{\prime}, u_{2}^{\prime}, \cdots, u_{\frac{n-1}{2}}^{\prime}$ be the vertices on the right side with respect to center $u_{0}$.

So for $P_{n}\left(P_{2}\right), V\left(P_{n}\left(P_{2}\right)\right)=V \cup V^{\prime} \cup U \cup U^{\prime}$, where

$$
\begin{aligned}
& V=\left\{v_{0}, v_{1}, v_{2}, \cdots, v_{\frac{\mathrm{n}-1}{2}}\right\}, \quad V^{\prime}=\left\{v_{0}, v_{1}^{\prime}, v_{2}^{\prime}, \cdots, v_{\frac{\mathrm{n}-1}{2}}^{\prime}\right\}, \\
& U=\left\{u_{0}, u_{1}, u_{2}, \cdots, u_{\frac{\mathrm{n}-1}{2}}\right\}, \quad U^{\prime}=\left\{u_{0}, u_{1}^{\prime}, u_{2}^{\prime}, \cdots, u_{\frac{\mathrm{n}-1}{2}}^{\prime}\right\} .
\end{aligned}
$$

CASE 2: For $P_{n}\left(P_{2}\right)$, when $n$ is even, let $v_{0}$ and $v_{0}^{\prime}, u_{0}$ and $u_{0}^{\prime}$ be the centers. Let $v_{1}, v_{2}, \cdots, v_{\frac{n}{2}-1}$ be the vertices on the left side with respect to center $v_{0}$ and $v_{1}^{\prime}, v_{2}^{\prime}, \cdots, v_{\frac{n}{2}-1}^{\prime}$ be the vertices on the right side with respect to center $v_{0}^{\prime}$ and $u_{1}, u_{2}, \cdots, u_{\frac{n}{2}-1}$ be the vertices on the left side with respect to center $u_{0}$ and $u_{1}^{\prime}, u_{2}^{\prime}, \cdots, u_{\frac{n}{2}-1}^{\prime}$ be the vertices on the right side with respect to center $u_{0}^{\prime}$.

So for $P_{n}\left(P_{2}\right), V\left(P_{n}\left(P_{2}\right)\right)=V \cup V^{\prime} \cap U \cap U^{\prime}$, where

$$
\begin{aligned}
& V=\left\{v_{0}, v_{1}, v_{2}, \cdots, v_{\frac{\mathrm{n}}{2}-1}\right\}, \quad V^{\prime}=\left\{v_{0}^{\prime}, v_{1}^{\prime}, v_{2}^{\prime}, \ldots, v_{\frac{\mathrm{n}}{2}-1}^{\prime}\right\}, \\
& U=\left\{u_{0}, u_{1}, u_{2}, \ldots, u_{\frac{\mathrm{n}}{2}-1}\right\}, \quad U^{\prime}=\left\{u_{0}^{\prime}, u_{1}^{\prime}, u_{2}^{\prime}, \cdots, u_{\frac{\mathrm{n}}{2}-1}^{\prime}\right\} .
\end{aligned}
$$

We say two vertices $u$ and $v$ are on opposite side in $P_{n}\left(P_{2}\right)$, if $u \in V \cup V^{\prime}$ and $v \in U \cup U^{\prime}$.

Definition 2.1. The level function $\ell$ from $V\left(P_{n}\left(P_{2}\right)\right)$ to set of nonnegative integers from a center vertex $c$ is defined as

$$
\ell(u):=\{d(u, c) ; c \text { is a center vertex }\} \text { for any } u \in V\left(P_{n}\left(P_{2}\right)\right) .
$$

Note that, in $P_{n}\left(P_{2}\right)$, the maximum level function is $\frac{n-1}{2}$ if $n$ is odd and $\frac{n}{2}-1$ if $n$ is even.

Observations. We made following observation for $P_{n}\left(P_{2}\right)$,

$$
\left|V\left(P_{n}\left(P_{2}\right)\right)\right|=2 n
$$

$$
d(u, v) \leq \begin{cases}\ell(u)+\ell(v), & \text { if } n \text { is odd } \\ \ell(u)+\ell(v)+1, & \text { if } n \text { is even. }\end{cases}
$$


Theorem 2.2. Let $P_{2}\left(P_{2}\right)$ be the cross product of $P_{2}$ and $P_{2}$. Then

$$
r n\left(P_{2}\left(P_{2}\right)\right)=4 .
$$

Proof. Radio labeling of $P_{2}\left(P_{2}\right)$ as shown in figure.

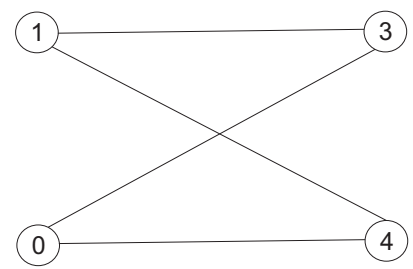

Figure 1: $r n\left(P_{2}\left(P_{2}\right)\right)=4$

Theorem 2.3. Let $P_{n}\left(P_{2}\right)$ be the cross product of $P_{n}$ and $P_{2}$ for $n \geq 3$. Then

$$
r n\left(P_{n}\left(P_{2}\right)\right) \geq n^{2}-n+1 .
$$

Moreover, the equality holds if and only if there exist a radio labeling $g$ with ordering $u_{1}, u_{2}, \ldots, n_{2 n}$ of vertices of $P_{n}\left(P_{2}\right)$ such that

$$
g\left(u_{1}\right)=0<g\left(u_{2}\right)<g\left(u_{3}\right)<\cdots<g\left(u_{2 n}\right),
$$

all the following holds, for all $1 \leq i \leq 2 n-1$,

(a) $u_{i}$ and $u_{i+1}$ are on opposite sides,

(b) $\left\{u_{1}, u_{2}\right\}=\left\{c_{1}, c_{2}\right\}$, where $c_{1}, c_{2}$ are center vertices.

Proof. Let $g$ be an optimal radio labeling for $P_{n}\left(P_{2}\right)$, where

$$
g\left(u_{1}\right)=0<g\left(u_{2}\right)<g\left(u_{3}\right)<\cdots<g\left(u_{2 n}\right) .
$$

Then

$$
g\left(u_{i+1}\right)-g\left(u_{i}\right) \geq(d+1)-d\left(u_{i}, u_{i+1}\right) \quad \text { for all } 1 \leq i \leq 2 n-1 .
$$

Summing these $2 n-1$ inequalities, we get

$$
r n\left(P_{n}\left(P_{2}\right)\right)=g\left(u_{2 n}\right) \geq(2 n-1)(d+1)-\sum_{i=1}^{2 n-1} d\left(u_{i}, u_{i+1}\right)
$$


Case (a): For $P_{n}\left(P_{2}\right)$, when $n$ is odd, we have

$$
\begin{aligned}
\sum_{i=1}^{2 n-1} d\left(u_{i}, u_{i+1}\right) & \leq \sum_{i=1}^{2 n-1}\left[\ell\left(u_{i}\right)+\ell\left(u_{i+1}\right)\right] \\
& =2 \sum_{u \in V(G)} \ell(u)-\ell\left(u_{1}\right)-\ell\left(u_{2 n}\right) \\
& \leq 2 \sum_{u \in V(G)} \ell(u) .
\end{aligned}
$$

Substituting (2.2) in (2.1), we get

$$
r n\left(P_{n}\left(P_{2}\right)\right)=g\left(u_{2 n}\right) \geq(2 n-1)(d+1)-2 \sum_{u \in V(G)} \ell(u),
$$

since $d=n-1$ and $\sum_{u \in V(G)} \ell(u)=\frac{n^{2}-1}{2}$, so

$$
r n\left(P_{n}\left(P_{2}\right)\right) \geq(2 n-1)(n)-2\left(\frac{n^{2}-1}{2}\right)=n^{2}-n+1 .
$$

CASE $(b)$ : For $P_{n}\left(P_{2}\right)$, when $n$ is even, we have

$$
\begin{aligned}
\sum_{i=1}^{2 n-1} d\left(u_{i}, u_{i+1}\right) & \leq \sum_{i=1}^{2 n-1}\left[\ell\left(u_{i}\right)+\ell\left(u_{i+1}\right)+1\right] \\
& =2 \sum_{u \in V(G)} \ell(u)-\ell\left(u_{1}\right)-\ell\left(u_{2 n}\right)+(2 n-1) \\
& \leq 2 \sum_{u \in V(G)} \ell(u)+(2 n-1) .
\end{aligned}
$$

Substituting (2.3) in (2.1), we get

$$
r n\left(P_{n}\left(P_{2}\right)\right)=g\left(u_{2 n}\right) \geq(2 n-1)(d+1)-2 \sum_{u \in V(G)} \ell(u)-(2 n-1),
$$

since $d=n-1$ and $\sum_{u \in V(G)} \ell(u)=\frac{n^{2}}{2}-n$, so

$$
r n\left(P_{n}\left(P_{2}\right)\right) \geq(2 n-1)(n)-2\left(\frac{n^{2}}{2}-n\right)-(2 n-1)=n^{2}-n+1 .
$$

Thus, from above two cases we have desired result. 
Theorem 2.4. Let $g$ be an assignment of distinct non-negative integers to $V\left(P_{n}\left(P_{2}\right)\right)$ and $\left\{u_{1}, u_{2}, u_{3}, \cdots, u_{2 n}\right\}$ be the ordering of $V\left(P_{n}\left(P_{2}\right)\right)$ such that $g\left(u_{i}\right)<g\left(u_{i+1}\right)$ defined by $g\left(u_{1}\right)=0$ and $g\left(u_{i+1}\right)=g\left(u_{i}\right)+d+1-d\left(u_{i}, u_{i+1}\right)$. Then $g$ is a radio labeling and for any $1 \leq i \leq 2 n-2$, the following holds.

$$
d\left(u_{i}, u_{i+1}\right) \leq \frac{n+1}{2} \text { if } n \text { is odd }
$$

(b) $\quad d\left(u_{i}, u_{i+1}\right) \leq \frac{n}{2}+1$ and $d\left(u_{i}, u_{i+1}\right) \neq d\left(u_{i+1}, u_{i+2}\right)$ if $n$ is even.

Proof. Let $g\left(u_{1}\right)=0$ and $g\left(u_{i+1}\right)=g\left(u_{i}\right)+d+1-d\left(u_{i}, u_{i+1}\right)$, for any $1 \leq i \leq 2 n-1$, and let for each $i=1,2, \ldots, 2 n-1, g_{i}=g\left(u_{i+1}\right)-g\left(u_{i}\right)$.

We want to prove that $g$ is a radio labeling, if $(a)$ and $(b)$ holds, that is, for any $j \neq i,\left|g\left(u_{j}\right)-g\left(u_{i}\right)\right| \geq d+1-d\left(u_{j}, u_{i}\right)$

CASE $(a)$ : When $n$ is odd, we have $d=n-1$ and let $(a)$ holds and we take $i>j$, then

$$
\begin{aligned}
& g\left(u_{i}\right)-g\left(u_{j}\right) \\
& =g_{j}+g_{j+1}+\cdots+g_{i-1} \\
& =(i-j)(d+1)-d\left(u_{j}, u_{j+1}\right)-d\left(u_{j+1}, u_{j+2}\right)-\cdots-d\left(u_{i-1}, u_{i}\right) \\
& \geq(i-j)(n)-(i-j)\left(\frac{n+1}{2}\right) \quad \text { by using }(1) \\
& =(i-j)\left(n-\left(\frac{n+1}{2}\right)\right) \\
& =(i-j)\left(\frac{n-1}{2}\right) \\
& \geq d+1-d\left(u_{i}, u_{j}\right) .
\end{aligned}
$$

CASE $(b)$ : When $n$ is even, let (b) holds and we take $i>j$

$$
\begin{aligned}
& g\left(u_{i}\right)-g\left(u_{j}\right) \\
& =g_{j}+g_{j+1}+\cdots+g_{i-1} \\
& =(i-j)(d+1)-d\left(u_{j}, u_{j+1}\right)-d\left(u_{j+1}, u_{j+2}\right)-\cdots-d\left(u_{i-1}, u_{i}\right)
\end{aligned}
$$


If $i-j=$ even, then

$$
\begin{aligned}
& g\left(u_{i}\right)-g\left(u_{j}\right) \\
& \geq(i-j)(d+1)-\frac{i-j}{2}\left(\frac{n}{2}+1\right)-\frac{i-j}{2}\left(\frac{n}{2}\right) \\
& =(i-j)(n)-(i-j)\left(\frac{n}{2}\right)-\frac{i-j}{2} \\
& =(i-j)\left(\frac{n}{2}\right)-\frac{i-j}{2} \\
& \geq d+1-d\left(u_{i}, u_{j}\right) .
\end{aligned}
$$

If $i-j=$ odd, then

$$
\begin{aligned}
& g\left(u_{i}\right)-g\left(u_{j}\right) \\
& \geq(i-j)(d+1)-\frac{i-j+1}{2}\left(\frac{n}{2}+1\right)-\frac{i-j+1}{2}\left(\frac{n}{2}\right) \\
& \geq d+1-d\left(u_{i}, u_{j}\right) .
\end{aligned}
$$

Thus, in both the cases $g$ is a radio labeling and hence the result.

Theorem 2.5. Let $P_{n}\left(P_{2}\right)$ be the cross product of $P_{n}$ and $P_{2}$ for $n \geq 3$. Then

$$
r n\left(P_{n}\left(P_{2}\right)\right) \leq n^{2}-n+1
$$

Proof. Here we consider following two cases.

CAsE 1: When $n$ is odd, define $g: V\left(P_{n}\left(P_{2}\right)\right) \rightarrow\left\{0,1,2, \ldots, n^{2}-n+1\right\}$ by $g\left(u_{i+1}\right)=g\left(u_{i}\right)+d+1-\ell\left(u_{i}\right)-\ell\left(u_{i+1}\right)$ as per ordering of vertices given below.

$$
\begin{aligned}
v_{0} \stackrel{\frac{\mathrm{n}-1}{2}}{\longrightarrow} v_{R k} \stackrel{\frac{\mathrm{n}+1}{2}}{\longrightarrow} v_{L 1} \stackrel{\frac{\mathrm{n}+1}{2}}{\longrightarrow} v_{R k} \stackrel{\frac{\mathrm{n}+1}{2}}{\longrightarrow} v_{L 1} \stackrel{\frac{\mathrm{n}-1}{2}}{\longrightarrow} v_{R(k-1)} \\
\stackrel{\frac{\mathrm{n}+1}{2}}{\longrightarrow} v_{L 2} \stackrel{\frac{\mathrm{n}+1}{2}}{\longrightarrow} v_{R(k-1)}^{\prime} \stackrel{\frac{\mathrm{n}+1}{2}}{\longrightarrow} v_{L 2}^{\prime} \stackrel{\frac{\mathrm{n}-1}{2}}{\longrightarrow} \cdots \stackrel{\frac{\mathrm{n}-1}{2}}{\longrightarrow} v_{R 1} \\
\stackrel{\frac{\mathrm{n}+1}{2}}{\longrightarrow} v_{L k} \stackrel{\frac{\mathrm{n}+1}{2}}{\longrightarrow} v_{R 1}^{\prime} \stackrel{\frac{\mathrm{n}+1}{2}}{\longrightarrow} v_{L k}^{\prime} \stackrel{\frac{\mathrm{n}-1}{2}}{\longrightarrow} v_{0}^{\prime}
\end{aligned}
$$

CASE 2: When $n$ is even, define $g: V\left(P_{n}\left(P_{2}\right)\right) \rightarrow\left\{0,1,2, \ldots, n^{2}-n+1\right\}$ by 
$g\left(u_{i+1}\right)=g\left(u_{i}\right)+d-\ell\left(u_{i}\right)-\ell\left(u_{i+1}\right)$ as per ordering of vertices given below.

$$
\begin{aligned}
v_{L 0} & \stackrel{\frac{n}{2}}{\longrightarrow} v_{R(k-1)} \stackrel{\frac{n+2}{2}}{\longrightarrow} v_{L 1} \stackrel{\frac{n}{2}}{\longrightarrow} v_{R(k-2)}^{\prime} \stackrel{\frac{n+2}{2}}{\longrightarrow} v_{L 2}^{\prime} \\
& \stackrel{\frac{n}{2}}{\longrightarrow} v_{R(k-3)} \stackrel{\stackrel{\frac{n+2}{2}}{\longrightarrow}}{\longrightarrow} \cdots \stackrel{\stackrel{\frac{n+2}{2}}{\longrightarrow}}{\longrightarrow} v_{L(k-1)} \stackrel{\frac{n}{2}}{\longrightarrow} v_{R 0} \stackrel{1}{\longrightarrow} v_{L 0}^{\prime} \\
& \stackrel{\frac{n}{2}}{\longrightarrow} v_{R(k-1)}^{\prime} \stackrel{\stackrel{n+2}{\longrightarrow}}{\longrightarrow} v_{L 1}^{\prime} \stackrel{\frac{n}{2}}{\longrightarrow} v_{R(k-2)}^{\prime} \stackrel{\frac{n+2}{2}}{\longrightarrow} v_{L 2}^{\prime} \stackrel{\frac{n}{2}}{\longrightarrow} v_{R(k-3)}^{\prime} \\
& \stackrel{\frac{n+2}{2}}{\longrightarrow} \cdots \stackrel{\frac{n+2}{2}}{\longrightarrow} v_{L(k-1)}^{\prime} \stackrel{\frac{n}{2}}{\longrightarrow} v_{R 0}^{\prime}
\end{aligned}
$$

Since $g$ satisfy conditions of Theorem 2.4, so $g$ is radio labeling with span $n^{2}-n+1$, hence

$$
r n\left(P_{n}\left(P_{2}\right)\right) \leq n^{2}-n+1 .
$$

Theorem 2.6. Let $P_{n}\left(P_{2}\right)$ be the cross product of $P_{n}$ and $P_{2}$. Then

$$
r n\left(P_{n}\left(P_{2}\right)\right)=n^{2}-n+1 .
$$

Proof. The proof follows from Theorem 2.3 and Theorem 2.5.

Example 2.7. In Figure 2, ordering of the vertices and optimal radio labeling of $P_{9}\left(P_{2}\right)$ is shown.

$$
\begin{aligned}
& v_{0} \longrightarrow v_{4}^{\prime} \longrightarrow v_{1} \longrightarrow u_{4}^{\prime} \longrightarrow u_{1} \longrightarrow v_{3}^{\prime} \longrightarrow v_{2} \longrightarrow u_{3}^{\prime} \longrightarrow u_{2} \\
& \quad \longrightarrow v_{2}^{\prime} \longrightarrow v_{3} \longrightarrow u_{2}^{\prime} \longrightarrow u_{3} \longrightarrow v_{1}^{\prime} \longrightarrow v_{4} \longrightarrow u_{1}^{\prime} \longrightarrow u_{4} \\
& \quad \longrightarrow u_{0} \\
& =\operatorname{rn}\left(P_{9}\left(P_{2}\right)\right) .
\end{aligned}
$$

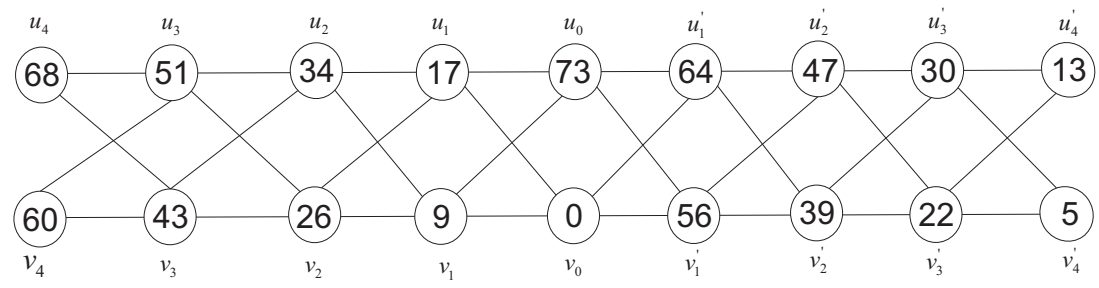

Figure 2: $r n\left(P_{9}\left(P_{2}\right)=73\right.$ 
Example 2.8. In Figure 3, ordering of the vertices and optimal radio labeling of $P_{10}\left(P_{2}\right)$ is shown

$$
\begin{aligned}
& v_{0} \longrightarrow v_{4}^{\prime} \longrightarrow v_{1} \longrightarrow v_{3}^{\prime} \longrightarrow v_{2} \longrightarrow v_{2}^{\prime} \longrightarrow v_{3} \longrightarrow v_{1}^{\prime} \longrightarrow v_{4} \\
& \quad \longrightarrow v_{0}^{\prime} \longrightarrow u_{0} \longrightarrow u_{4}^{\prime} \longrightarrow u_{1} \longrightarrow u_{3}^{\prime} \longrightarrow v_{2} \longrightarrow u_{2}^{\prime} \longrightarrow u_{3} \\
& \quad \longrightarrow u_{1}^{\prime} \longrightarrow u_{4} \longrightarrow u_{0}^{\prime} \\
& =\operatorname{rn}\left(P_{10}\left(P_{2}\right)\right)
\end{aligned}
$$

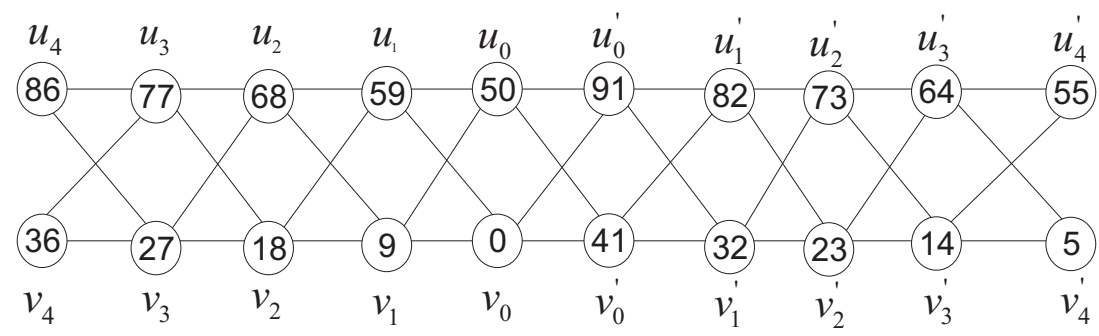

Figure 3: $r n\left(P_{10}\left(P_{2}\right)\right)=91$

\section{References}

[1] G.J. Chang, D. Kuo, The $L(2,1)$-labeling problem on graphs, SIAM J. Discrete Math., 9 (1996), 309-316.

[2] G. Chartrand, D. Erwin, P. Zhang, F. Harary, Radio labelings of graphs, Bull. Inst. Combin. Appl., 33 (2001), 77-85.

[3] J.P. Georges, D.W. Mauro, Labeling trees with condition at distance two, Discrete Math., 269 (2003), 127-148, doi: 10.1016/S0012-365X(02)007501

[4] J.R. Griggs, R.K. Yeh, Labeling graphs with condition at distance 2, SIAM J. Discrete Math., 5 (1992), 586-595.

[5] W.K. Hale, Frequency assignement: theory and application, Proc. IEEE, 68 (1980), 1497-1514.

[6] D.D.F. Liu, Radio number for trees, Discrete Math., 308 (2008), 11531164, doi: 10.1016/j.disc.2007.03.066 
[7] D.D.F. Liu, M. Xie, Radio number for square cycles, Congr. Numer., 169 (2004), 105-125.

[8] D.D.F. Liu, M. Xie, Radio number for square paths, Ars Combin., 90 (2009), 307-319.

[9] D.D.F. Liu, X. Zhu, Multi-level distance labeling for paths and cycles, SIAM J. Discrete Math., 19 (2005), 610-621.

[10] P. Martinez, J. Ortiz, M. Tomova, C. Wyels, Radio numbers for generalized prism graphs, Discuss Math. Graph Theory, 31 (2011), 45-62.

[11] M.T. Rahim, M. Farooq, M. Ali, S. Jan, Multi-level distance labelings for generalized gear graph, Int. J. Math. Soft Comput., 2 (2012), 57-63.

[12] F.S. Roberts, T-coloring of graphs: recent results and open problems, Discrete Math., 93 (1991), 229-245, doi: 10.1016/0012-365X(91)90258-4

[13] D. Sakai, Labeling chordal: distance two condition, SIAM J. Discrete Math., 7 (1994), 133-140.

[14] S.K. Vaidya, D.D. Bantva, Labeling cacti with a condition at distance two, Le Matematiche, 66 (2011), 29-36.

[15] S.K. Vaidya, P.L. Vihol, Radio labeling for some cycle related graphs, Int. J. Math. Soft Comput., 2 (2012), 11-24.

[16] S.K. Vaidya, P.L. Vihol, N.A. Dani, D.D. Bantva, $L(2,1)$-labeling in the contexamplet of some graph operations, J. Math. Res., 2 (2010), 109-119.

[17] W.F. Wang, The L(2,1)-labeling of trees, Discrete Appl. Math., 154 (2006), 598-603, doi: 10.1016/j.dam.2005.09.007

[18] R.K. Yeh, Labeling Graphs with a Condition at Distance Two, Ph.D. Thesis, Department of Mathematics, Universoty of South Carolina, Columbia, South Carolina, 1990.

[19] R.K. Yeh, A survey on labeling graphs with condition at distance two, Discrete Math., 306 (2006), 1217-1231, doi: 10.1016/j.disc.2005.11.029 
